\title{
Wireless Body Area Networks: Survey of recent research trends on energy efficient routing protocols and guidelines
}

Shunmugapriya B ( $\sim$ priyalakshminarayanan512@gmail.com )

National Engineering College Department of Computer Science and Engineering https://orcid.org/0000-0001-8166-4586

Paramasivan B

National Engineering College Department of Mechanical Engineering

Ananthakumaran S

KL University

Naskath J

National Engineering College

\section{Research Article}

Keywords: Energy-Efficient, WBAN, Survey, Routing Protocols, Network Lifetime.

Posted Date: July 22nd, 2021

DOI: https://doi.org/10.21203/rs.3.rs-385025/v1

License: (9) This work is licensed under a Creative Commons Attribution 4.0 International License. Read Full License 


\title{
Wireless Body Area Networks: Survey of recent research trends on energy efficient routing protocols and guidelines
}

\author{
B.Shunmugapriya ${ }^{1}$, Dr.B.Paramasivan ${ }^{2}$, Dr.S.Ananthakumaran ${ }^{3}$, J.Naskath ${ }^{4}$ \\ ${ }^{1,4}$ Department of Computer Science and Engineering \\ ${ }^{2}$ Department of Information Technology \\ National Engineering College, Kovilpatti, Tamilnadu, India \\ ${ }^{3}$ Department of Computer Science and Engineering \\ KL University, Vijayawada, Andhrapradesh, India
}

\begin{abstract}
:
Wireless Body Area Network (WBAN) is a recent technologically advanced sub-category of Wireless Sensor Networks (WSN) which consists of many bio-sensors, attached in and around of human body, for monitoring the health-related issues from remote places. Energy conservation in WBAN has always been the most crucial issue for the sensor nodes, which are powered by limited capacity battery sources. During sensing and communication, sensing elements emit high temperature from its circuit and transmitting antenna due to inefficient data routing designs. This energy may lead to form hot-spot, which affects the human tissues at a different level. So designing an energy-efficient routing protocol is a very challenging issue to reduce the high emission of temperatures. Different energy-efficient routing protocols have been projected in this survey over the years. The energy-efficient routing protocols endeavor to prolong the network lifetime by minimizing the energy consumption in each deployed nodes. There have been a variety of survey papers put forwarded by researchers to evaluate the performance and categorize the different energy-efficient routing protocols for WBANs. This paper describes an overview of WBAN, a systematic survey of existing contracts for routing and open research issues are discussed.
\end{abstract}

Keywords: Energy-Efficient, WBAN, Survey, Routing Protocols, Network Lifetime.

\section{Introduction}

According to statistics from World Population scenario: the 2017 Review, the number of older persons - those aged 60 years or higher - is expected to above twice by 2050 and above triple by 2100, rising from 962 million internationally in 2017 to 201 billion in 2050 and 3.1 billion in 2100 . Worldwide, the people aged 60 or above are increasing faster than all younger age groups. Universally, the number of persons aged 80 or more over is projected to three times by 2050, from 137 million in 2017 to nearly 425 million in 2050. By 2100 it is expected to raise to 909 million, almost seven times its value in 2017 [33]. Since the aged people belonging to this age group are going to take the risk with different types of health problems, they may require more frequent medical treatment to survive in the world. So, it is inconvenient for them if they want to move for a long distance from home place to the medical center and also periodic medical checkup is costly. Therefore, the research group concerning this issue takes as a serious concern with limited resources.

At present, in most of the regions in the world with economically forwarded and back- wording countries, traditional medical care is following, and these caring centers are monitoring the patients at a particular time of a day or a week. This style of diagnosis process is not yielding fruitful results to reduce their health issue. Therefore, a continuous patient monitoring system is needed to achieve better treatment for this age group of patients.

In the 21st century, rapid growth and advances in the development of microelectronics and micro-electro-mechanical systems (MEMS), small and smart devices have been found out. The components of this device or sensor structure are shown in Fig. 1.

Sensors are playing a important role in the day to day life like home automation, precision agriculture, inventory, remote monitoring, intelligent transportation system, sports, entertainment, and military, etc. Distributed sensor nodes, having a wireless transceiver, and communicate wirelessly to transmit the data, are referred to as a Wireless Sensor Network (WSN). With more than one battery-powered devices can be attached to the patient on/in the body or on the clothes to monitor a different kind of bio-signals of the person. These signals are categorized like ECG, EEG, blood pressure, glucose level, etc. Networking all such types of sensors is called Wireless Body Area Networks (WBAN).

Although WBAN is the sub-type of WSN, WBAN is differed from some WSN parameters and unable to implement all the notion of the protocol of WSN. Hence, the WBAN monitoring system is restricted to the human body. The bio-compatible sensor devices and more variable network topologies are the most required for recent years due to body movement. The main issues and challenges of WBAN are deploying the sensor nodes, the mobility of nodes during movement of patients, data storage, battery life or usage of energy efficiency, and security. The effectiveness of WBAN is defined by two critical parameters, namely energy consumption over the lifetime of the system and the network throughput. Energy is a significant input for the economic and societal development of WBAN in recent years. Since WBAN routing protocols are power-hungry, most of the strength of the battery is consumed during the data transmission phase.

Several routing protocols have been proposed till now for the applications of WBAN, and these protocols are classified with respect to their intention. The first category is cluster-based routing protocols in which the network is divided into a cluster of nodes formed a group; each cluster has a cluster head responsible for collecting the sensed data from its cluster and forwarding it to the base station. The main goal of this type of protocol is that minimizing direct communication between sensor nodes and the sink nodes. The second class is energy-efficient routing protocols, which are mainly focusing on reducing the temperature level 
of sensor nodes in the network during the data transmission period. Cross layered based protocols is the third level of BAN routing protocols mainly concerned about the routing with medium access issues. The fourth category of routing protocols is QoS based, which are focusing on higher reliability, low end-end delay, and higher packet delivery ratio. A postural movement-based routing protocol is the fifth in a row, mainly worried about the movement of sensor nodes' positions and thereby changing the distance between nodes and sink. Here, the action of the nodes is taken care of, and hence the energy consumption of this event is raised considerably. So, the network lifetime is decreased. The sixth category is the cost-effective routing protocol, which updates the cost feature based on appropriate data and finds its path between routes with minimal costs. The last class in this row is the secure routing protocol, which are primary concerns about the safety of data during the transmission period. Since WBAN is most applied in the health sector, the protection of information during transmission and in the database is essential. Leaking health-related data related to one patient leads to disastrous. All these types of routing protocols are tries to satisfy a particular objective of WBAN. This study has explored different proposed routing protocols to meet specific goals and requirements of WBANs.

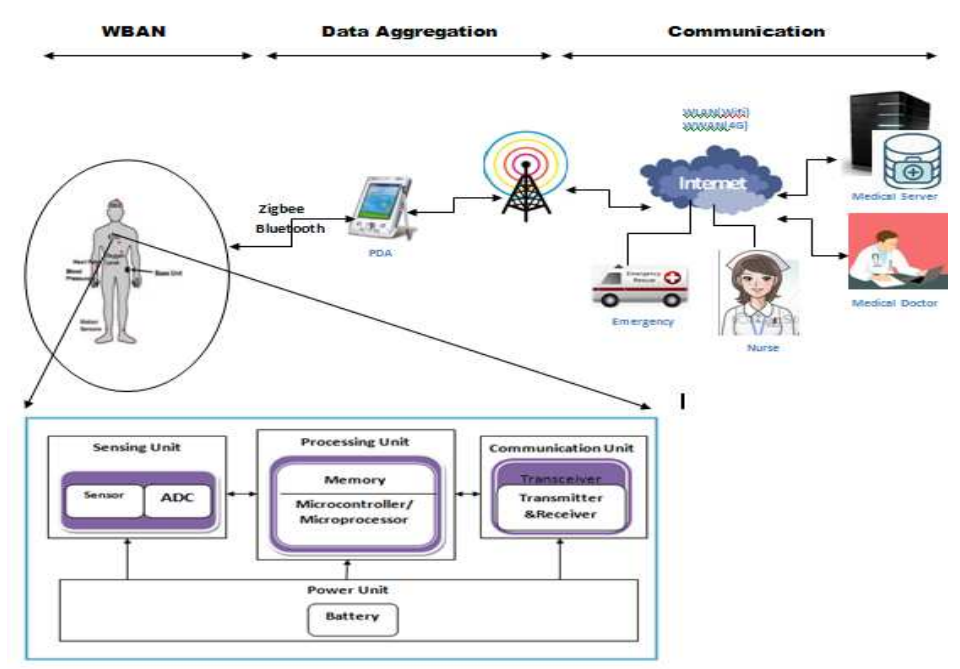

Fig. 1: BAN Architecture and Components of Sensor

2. Application of WBAN with Energy Efficient Routing Protocol

Application of WBAN is essential in society compared to other networking applications. It is mainly categorized into two types, as shown in Fig. 2. They are

- Medical application

- $\quad$ Non-Medical Applications

The utilization of a Wireless Body Area Network (WBAN) is limited, but its role is vital in the life-saving of the human being. So, WBAN finds its use mostly in the medical and healthcare systems. Hence a lot of development progress and research is going in this area to broaden its application assortment. The following are the main applications of WBAN: Telemedicine and remote patient monitoring, Rehabilitation and therapy, Bio-feedback, and Ambient Assisted Living. There are different kinds of technologies incorporated with WBAN for its application at a different level. Some of the techniques in WBAN are Bluetooth, Zigbee, IEEE803, Ultra-Wideband (UWB) technology, ANT protocol, and Zarlink technology. 


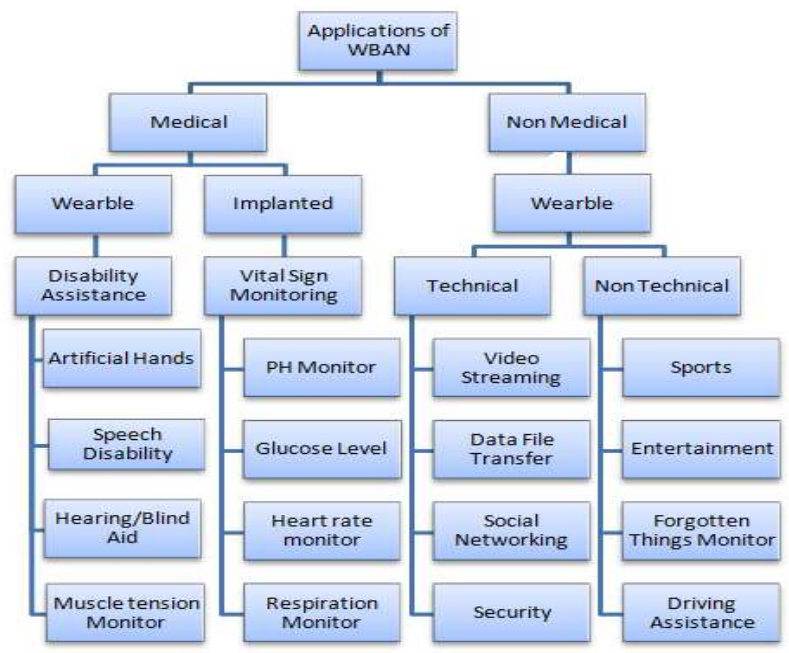

Fig. 2: Applications of WBAN

\section{Mathematical Modeling}

The formulation of a problem requires an analysis of the proposed system. This analysis will show various phases and the way it can be controlled.

\subsection{Integer Linear Programming Model}

To construct a model in which effectiveness of the system is expressed as a function of the variables defining the system. The general form of the model can be represented as follows:

$$
E=f\left(x_{i}, y_{j}\right)
$$

Where

$\mathrm{E}=$ Effectiveness of the system

$x_{i}=$ Variables of the system that can be controlled

$y_{j}=$ variables of the system that cannot be controlled but do not affect $\mathrm{E}$.

Deriving a solution from such a model consists of determining those values of control variables $x_{i}$, for which the measure of effectiveness is optimized. Optimization includes both maximization and minimization.

In order to optimize energy consumption minimization [34] and throughput and network lifetime maximization [13], linear programming based mathematical models are proposed.

The reason for choosing linear programming models is (a) to attain optimum use of productive factor, (b) more objective than subjective, (c) better for adjustments to meet changing conditions, (d) allows modification of its mathematical solutions, (e) highlights the bottlenecks in the processes.

Let us consider the subsequent general Integer linear programming (LP) representation:

$$
\begin{gathered}
\text { Maximize/Minimize } Z=\sum_{j=1}^{n} c_{j} x_{j}, \\
\text { Subject to } \sum_{j=1}^{n} a_{i j} x_{j} \leq b_{i},\left(b_{i} \geq 0\right) \quad i=1,2,3, \ldots, m \\
x_{j} \geq 0, \quad j=1,2,3, \ldots, n
\end{gathered}
$$


Where variables $x_{j}(j=1,2,3, \ldots, n)$ are called decision variables, $c_{j}, a_{i j}$ and $b_{i}(i=1,2,3, \ldots, m) ; j=(1,2,3, \ldots, n)$ are constants determined from the definition of the problem. The constants $c_{j}$ are called objective function coefficients, and constants $b_{i}$ are called stipulations and constants $a_{i j}$ are called structural coefficients. In general in Linear Programming (LP) $m<n$.

\subsubsection{Maximum Throughput Model}

In WBAN, each bit of information should be taken care of; otherwise, it leads to missing a few data related to patients. Thus the objective is to increase successful packet reception at the sink. The authors [34] proposed a model for collecting data by forwarder node from its member nodes and then forward it to the sink. [9] Formulated the following Integer Linear Programming models discussed on [34] aims to maximize the number of successfully received packets at the sink throughout the network lifetime.

Maximize $\sum_{r} P_{s}, \forall r \in R$

Subject to

$$
\begin{aligned}
& A_{i f}=A_{f s}=1, \forall i, f \in N \\
& P_{i f}=P_{f s}=0, \forall i, f \in N \\
& E_{i} \geq E_{\min }, \forall i \in N \\
& p_{l} \geq p_{\min }, \forall l \in L \\
& \sum_{i} \lambda_{i} t \leq c b_{i}, \forall i \in N
\end{aligned}
$$

In this objective function, constraint 1 fulfills the essential requirement that is all the links are established. Violation of restrictions 2-4 leads to packets being dropped, which ultimately leads to decreased throughput. Violation of restriction 5 leads to packets arrival beyond the handling capacity, causing reduced throughput as well as increased delay.

\subsubsection{Minimum Energy Consumption Model}

In [9] formulated the following Integer Linear Programming model to minimize energy consumption based on the optimal solution designed on [34]. Here two different types of nodes, forwarder and regular, are considered. Data collection from other normal nodes, data aggregation, and transmit the aggregated data to sink are done by the forwarder node. Each of these tasks consumes different energy. Sending energy of the forwarder node depends on the amount of data collected from other nodes, distance from the sink, and aggregation of the received data. So, the energy is consumed in two ways: Data Transmission and Data Reception. (a).Forwarder node data transmission,

(b). Normal node data transmission,

(c). Data transmission of all nodes during entire network lifetime,

(d). Data reception by forwarder node,

(e). Data reception for the entire network life time.

Assume that $A_{i f}$ represents a link between normal node and forwarder node. Similarly, $A_{f s}$ represents a link between forwarder and sinks. 
$F_{i f}^{s}$ represents traffic flow between a node and forwarder. Also, $F_{f s}^{t}$ represents the total traffic flow between forwarder node and sink.

$\operatorname{Minimize}\left(\sum_{i \in N} E_{i}+E_{f}\right) \forall i, f \in N$

Where

$E_{i}=A_{i f}\left(E_{T x-e l e c}+\epsilon_{a m p} n d_{i f}^{n}\right)$, energy consumption of sensor nodes

$E_{f}=\sum A_{i f}\left(E_{R x-e l e x}\right)+E_{d a}+A_{f s}\left(E_{T x-e l e c}+\in_{a m p} n d_{f s}^{n}\right)$, energy consumption of forwarder node

Subject to

$$
\begin{aligned}
& E_{i} \leq E_{0} \quad \forall i \in N \\
& E_{f} \leq E_{0} \quad \forall f \in N \\
& \sum_{i} F_{i f}^{t} \leq C_{i f} \quad \forall i \in N \\
& F_{f s}^{t} \leq C_{i f} \forall f \in N \\
& \sum_{i} b_{i f}+\lambda_{f} t \geq b_{f s}^{t} \quad \forall i, f \in N \\
& d_{i f} \wedge d_{f s} \rightarrow d_{\min }
\end{aligned}
$$

Constraints 1 and 2 represent the energy capacity constraints or normal node and forwarder node. Constraints 3 and 4 state that the total flow of data with their physical link capacities. Violating these two constraints leads to high energy consumption. Constraint 5 guarantees that the flow conservation with parameters of length of data and data generation rate at forwarder node at a time. Constraint 6 warns us that the communication distance needs to be minimized.

\subsubsection{Network Lifetime}

Consider there are $\mathrm{n}$ different number of sensor nodes and $\left(\mathrm{n}+1^{\text {th }}\right.$ node is as Sink. The locations of every node know each other by broadcasting HELLO message. Also consider network lifetime R rounds and let $P_{i, j}$ be the total number of packets that node $i$ transmits to node $j$, during round $r$. The objective function to achieve maximum network lifetime is as follows:

Maximize $R=\sum_{i} r$

Subject to

$$
\begin{aligned}
& C_{1}: \sum_{i, j}\left(P_{i, j} \times E_{i, j}^{t x}\right)+\sum_{j, i}\left(P_{j, i} \times E_{j, i}^{r x}\right) \leq E_{i} \forall i \in n, \\
& C_{2}: \min _{i} T x_{i}^{r e} \\
& \forall i \in n,
\end{aligned}
$$




$$
\begin{aligned}
& C_{3}: \min \sum_{i} L_{i}^{u n} \quad \forall i \in n \\
& C_{4}: \min \sum_{i} d_{i, j}^{n+1} \forall i, j \in n, \\
& C_{5}: \sum_{i, j} \gamma_{i, j}+\sum_{i}\left(\Gamma_{i} \times t_{r}\right) \leq \sum_{j, i} \gamma_{j, i} \quad \forall i, j \in n, \\
& C_{6}: 0 \leq \gamma_{i, j} \leq P_{i, j} \quad \forall i, j \in n, \\
& C_{7}: \sum_{i} \gamma_{i, n+1}=T \quad \forall i \in n
\end{aligned}
$$

Constraint 1 guarantees that the available energy of the node $E_{i}$ is respected by the link capacities. Constraint 2 ensures minimizing packet retransmissions, which results in a long network lifetime. Constraint 3 notes that the wasteful transfer of data over the lifespan of the network is minimized. Constraint 4 implies the direct relationship between the distance of contact and the lifespan of the network. Constraint 5 indicates that the data flow should not surpass the outgoing flow of the generated data; otherwise, loss or retransmission of the data packet will lead to more energy consumption. Constraint 6 , instead, guarantees the capacity of the flow network links. $\quad \sum_{i, j} \gamma_{i, j}=\sum_{i, j} \gamma_{j, i}$

Constraint 7 informs us that capacity on the links/edges of the flow network must be maintained.

\subsection{Path Loss Model}

In general, Signal attenuation is called Path Loss. Otherwise, it can be defined as the difference between the transmitted power and received power where antenna gain may or may not be considered. In WBANs, the objects movement inside the body and outside the body may affect the transmitted signal. As far as concern in WBANs, path loss depends on distance and frequency. The path loss model (in $\mathrm{dB}$ ) as a function of distance between transmitter and receiver is analyzed in [23] using the following equation.

$$
P L=P L_{0}+10 n \log _{10}\left(\frac{d}{d_{0}}\right)+\sigma_{s}
$$

Where

$P L_{0}$ is path loss at reference distance $d_{0}$

The path loss exponent is $\mathrm{n}$ (for space its value is 2 and for human body its value varies from 4 to 7 )

D is the distance from transmitter to receiver ${ }^{\sigma} s$ is standard deviation

In WBANs path loss may happen due to free space loss, reflection, refraction and diffraction. By having such disturbance, the transmitting / transmitted signals' frequency may be in weak. This path loss could be analyzed on [23] using the following equation. 


$$
P L_{0}=10 \log _{10}\left(\frac{4 \pi d}{\lambda}\right)^{2}
$$

or

$$
P L_{0}=10 \log _{10}\left(\frac{4 \pi d f}{c}\right)^{2}
$$

Where, $f$ is frequency of the propagating wave, $\lambda$ is wave-length of the propagating wave, and $c$ is the speed of light. Most of papers related to WBANs used operating frequency of $2.4 \mathrm{GHz}$ ISM band.

\subsection{Radio Model}

Many radio models have been proposed for mode of communication in the literature. The amount of energy consumed depends on the modes of communication used. In WBAN, data packets can be transmitted in two ways: (a) Transmit emergency data directly to the Sink node using Single-hop communication, (b) Transmit the data in normal way using Single-hop or Multihop mode of communication.

\subsubsection{Single-hop Communication Model}

The energy consumption during single-hop communication is calculated on [10] as follows:

$E_{s-h o p}=E_{t x} \quad$ Where, $E_{t x}$ is transmission energy.

$$
E_{t x}=E_{\text {elec }}+E_{\text {amp }}
$$

Where $E_{\text {elec }}$ is the energy consumed for processing data and $E_{a m p}$ is the energy consumed by transmit amplifier.

$$
E_{s-h o p}=E_{t x}=b \times\left(E_{e l e c}+E_{a m p}\right) \times d^{2}
$$

Where $d^{2}$ is energy loss due to the transmission or the distance between transmitter node to receiving node.

Usually, receiving, processing, and transmission of data on each intermediate node take much time, which causes delays. Sometimes, this delay is also augmented due to congestion and becomes intolerable in some severe scenarios. So, single-hop communication is used to reduce this setback. In single-hop communication, the sensor node uses the full power of the battery to send its data directly to the Sink node. Due to this direct communication, the delay can be reduced and can be useful to take of the patients as quickly as possible.

\subsubsection{Multi-hop Communication Model}

The energy consumption during Multi-hop communication is calculated on [10] as follows:

$E_{m-h o p}=E_{t x}+E_{r x}$

where $E_{r x}$ is the energy consumed for receiving data. If the system is transmitting $b$-bits to a distance of $n$-hops the transmission energy will be $n \times b \times E_{t x}$ and receiving energy will be $(n-1) \times b \times E_{r x}$. Since the initial node transmits only and an intermediary nodes receive $n$-bits and then transmit these received bits. So the energy consumed for Multi-hop is: 


$$
E_{m-h o p}=\left(n \times b \times E_{t x}\right)+\left((n-1) \times b \times E_{r x}\right)
$$

$E_{r x}=E_{\text {elec }}$, Given that the receiving energy is equivalent to the energy consumed to process received data. Then the previous equation can be written as:

$$
\begin{aligned}
& E_{m-h o p}=\left((n \times b) \times\left(E_{\text {elec }}+E_{\text {amp }}\right) \times d^{2}\right)+\left((n-1) \times b \times E_{\text {elec }}\right) \\
& \left.E_{m-h o p}=\left((n \times b) \times E_{\text {elec }}\right)+\left((n \times b) \times E_{\text {amp }} \times d^{2}\right)+\left((n \times b) \times E_{\text {elec }}\right)-b \times E_{\text {elec }}\right) \\
& E_{m-h o p}=\left(2 \times(n \times b) \times E_{\text {elec }}\right)+\left((n \times b) \times E_{\text {amp }} \times d^{2}\right)-\left(b \times E_{\text {elec }}\right)
\end{aligned}
$$

\subsubsection{Cost Function}

Multi-hop communication model is preferred to minimize energy consumption and maximize throughput in WBAN. A forwarder node has to be selected to balance the energy. This selection can be done by calculating the cost function of all nodes. Suppose $i$ is the identification of a node then the cost function is computed as follows:

$$
C F_{i}=\frac{d_{i}}{R E_{i}}
$$

Where $d_{i}$ is the distance between the node, $i$ and sink, and $R E_{i}$ is the residual energy of node $i . R E_{i}$ is calculated by subtracting the presently consumed energy from previously available total energy. Hence, the forwarder node has minimum $d_{i}$ and maximum $R E_{i}$.

\subsubsection{Radiation Energy Analysis}

Radio signals generated through wireless communication generate electromagnetic fields, which is absorption of human tissue leading to temperature rise. The amount of radiation energy absorbed by human tissue is referred as the Specific Absorption Rate (SAR) [29].

$$
S A R=\frac{\sigma|E|^{2}}{\rho}(W / K g),
$$

where $\sigma$ is the electrical conductivity of tissue, $E$ is the electric field induced by radiation and $\rho$ is the density of tissue. The objective of the SAR is to maintain a low temperature to avoid routing on hot spot. The result referred in [29] is that SAR of 8 $\mathrm{W} / \mathrm{Kg}$ for 15 minutes can cause significant tissue damage. The norm of the Federal Communications Commission (FCC) states that the upper limit of SAR for near-field exposure is $1.6 \mathrm{~W} / \mathrm{Kg}$ for any tissue averaged over one gram, while the upper limit of 2.0 W/Kg for $10 \mathrm{~g}$ of tissue is specified by the International Commission on Non-Ionizing Radiation Safety (ICNIRP). The analysis in[29] converts these SAR values into a rise in temperature, and the maximum values for potential human head and brain temperature changes are $0.31 \mathrm{oC}$ and $0.13 \mathrm{oC}$ for the FCC standard and $0.60 \mathrm{oC}$ and $0.25 \mathrm{oC}$ for the ICNIRP standard. The study in [29] also shows that a temperature increase of $0.10 \mathrm{C}$ is sufficiently high to cause an extreme thermoregulatory response from the human body.

\section{Terminologies Used in WBAN}


In this section, this paper explains the terms generally used in the development of WBAN. Most importantly, some of these terms are used to classify the surveyed energy-efficient routing protocols in succeeding sections.

\subsection{Network Lifetime}

It is one of the vital assessments metric that is mostly used to evaluate the energy efficiency of WBAN. The key reason to propose a energy efficient routing protocol for WBAN is to increase the network lifetime. In a WSN, the network lifetime generally defined as the maximum duration of time that a Wireless body area Network would be fully active. Or other the time duration between the network boot / renew to the end when the final sensor nodes or the bulk of sensor nodes expire. The collapse of any one sensor node may lead to network breakdown. Each and every node of the network is very important for increasing the energy efficiency of WBAN.

\subsection{Throughput}

The general definition of throughput is a calculate of how many units of information a system can process in a given time amount or other the rate of successful message deliverance over a communication channel.

\subsection{Residual Energy}

Its energy level generally measures the life span of a sensor nodes after a given network data transmission round. Usually, the energy level in sensor nodes after every network data transmission phase is referred to as its residual energy. When improving the network performance by the best possible use of residual energy and enhancing network lifetime. The residual energy

$$
\begin{aligned}
& \text { of sensor } \\
& \text { node } \\
& \text { can } \\
& \text { be } \\
& \text { calculated } \\
& E \text { (residual energy) }=E \text { (initial deployment) } \sum_{\text {initial round }=0}^{\text {Total number of rounds }}-E \text { (data transmission round) }
\end{aligned}
$$

as:

\subsection{Path loss}

In WBAN, the sensor nodes are placed in different places of the human body. Path loss is an attenuation of a transmitted signal for source to destination. Path loss may measure in free space and on-body associations for bodies with considerably different physical structures. When WBAN wants to increase the performance, channel characterization and representation are required by revise path loss characteristics of the channels. The path loss is dependent on frequency as well as the distance of the transmitter to the receiver side. If the medium is the line of sight, then the signal achieves very minimum attenuation. But WBAN is dealing with the human body, and it is not a line of sight, messages may have some mitigation. The path loss component in the human body varies from 4 to 7 and in free space are 2.

\subsection{Packet Delivery Ratio}

Since this paper is dealing with WBAN, packet delivery ratio (PDR) is very important. The medical data must be reached fully to the intended destination place within a certain period of time, or otherwise, the sent data is useless. Packet delivery ratio (PDR) is that by several packets obtained at the sink node divided by the number of packets sent from the source.

\section{Related Works}

An Energy Efficiency Routing Protocol for Wireless Body Area Sensor Networks: This clustering based routing protocol [5] uses multi-hop communication model for wireless body area network (CRPBA) guaranteed for optimizing the energy consumption by a sensor nodes during data transmission and through which this method increases the network lifetime and network stability.

In this work, the authors have deployed 24 different sensors and 2 different gateway body sensors are organized on the human body to gather the bio-signals and transmit to the base station respectively. The base station is placed outside of the network. Here, emergency data are transmitted directly to the gateway and the normal data are transmitted to the gateway through clustering algorithm.

In order to save the energy, special cluster head is selected for each round with minimum distance of gateway and maximum energy. The selection of cluster head is done by using the following equation:

$$
\text { Function }(D, E)=\text { Distance/Energy; }
$$

So, the cluster head collecting data from cluster members, combined it and forwarding to the gateway of the body sensor, which in turn directs data to the base station. Since maximum energy is wasted during transmission and reception of data, combine the collected data from different sensors to reduce number of transmission and avoid redundant transmission. The energy consumption model is as follows:

$$
E_{s-h o p}=E_{t x}=b \times\left(E_{\text {elec }}+E_{\text {amp }}\right) \times d^{2}
$$

Where $d^{2}$ is energy loss due to the transmission or the distance between transmitter nodes to receiving node. The energy for receiving data is calculated as follows:

$$
E_{r x}=b \times\left(E_{e l e c}\right)
$$


The first node dies at $3375^{\text {th }}$ round when CRPBA is used and at $2611^{\text {th }}$ round when CRPBA is not used. Since each node consumes reasonable energy which helps to have more stability period and longer network lifetime. Thus, as a result of this work is demonstrated that CRPBA protocol consumes minimum energy till $60 \%$ of simulation time.

The major advantage of CRPBA protocol has changing cluster head, with minimum distance and maximum energy, for each round to maintain the energy balance. However, throughput, reliability and energy efficiency were not optimized properly in view of the fact that due to stringent constraints in computational power and energy.

An Energy Efficient Routing Protocol for Wireless Body Area Sensor Networks: This is a single-hop and multihop communication routing protocol to achieve the highest network stability, good throughput with the availability of sensor node, maintaining average energy consumption, and producing less path loss.

In this proposed work [24], a total 8 different sensor nodes are deployed, and 2 of them are sending emergency data

directly to the sink node, are not part of the multi-hopping communication model. The forwarding node concept has been introduced for the remaining six different sensor nodes. The authors used a mathematical model to calculate the consumption of energy for sensing data, processing the collected data, and transmitting the processed data to the sink is as follows:

$$
E_{t x}=b \times\left(E_{\text {elec }}+E_{\text {amp }}\right) \times d^{2} \text {. }
$$

The amount of energy consumed by a sensor node in WBAN during transmission is as follows:

$$
E_{\text {node }}=E_{t x}+E_{\text {retx }}+E_{a c k}+E_{a c c}
$$

where the parameters represent transmission energy, retransmission energy, energy consumed while transmitting acknowledgment packet, and power used by the channel access procedure, respectively. In this model, the Nordic nRF2401A transceiver is used because it consumes less power. Hence, it will extend the network lifetime.

The cost function mentioned in equation (25) is followed to find out the forwarding node for gathering data from sensor nodes. The communication between sensor nodes and forwarding node is based on Time Division Multiple Access (TDMA), which gives time slots for nodes to send observed data.

This protocol is compared with the M-ATTEMPT protocol. The network lifetime and network stability are based on the life of nodes. Since this work used Nordic nRF2401A transceiver, the sensors spend less energy for data transmission. So, the residual power is maintained, and the lifetime of the sensor node is extended. Therefore, the network stability is strengthened, and through which lifetime of the network is trivially increased. Due to the sensor nodes' lifetime is increased, the throughput is also increased with the total number of packet received as 3.25 x 104. The path loss of the proposed work is less because the live nodes of the proposed network are large. This work achieves the energy consumption with the help of forwarder cost function in every round.

The advantage of this work is forwarding node selection using a cost function. This selection is used to balance the energy of all available nodes, and it leads to an increase in the lifetime of the network.

This protocol uses only six nodes to sense the bio-signals and transmit it to the nearest sink node. Since the number of nodes in the network is very less, the distance between different nodes is a concern. If the sink is nearby, then transmission takes less energy. On the other hand, suppose the sink is far away from the transmitting sensor, it takes more energy. Mostly WBAN concerns about patients' information and that should be taken in care, safe, and dangerous approach. Moreover, data is traveling with the long-distance the security of data is a concern in this point of view. So, safe and secure WBAN architectural design is urgently required at this juncture.

Also, M-ATTEMPT addresses and supports the mobility of the sensor nodes deployed on the human body, but this energyefficient routing protocol is discussed only about fixed nodes.

RE-ATTEMPT: A new energy-efficient routing protocol for WBAN: This protocol [13] fixed the wireless sensors on the human body according to energy levels. Here, single-hop communication is used for emergency data and multi-hop communication for normal data delivery. Routing with minimum hop count is selected for data delivery. The major intention of this work is to increase network life time, throughput and decrease the packet drop ratio. This protocol deals with linear integer programming model for maximization of network life time. 
i. Initialization Phase: In this phase three different works have been executed as follows: (a) each node is informed with its neighbor, (b) position of sink, (c) all possible routes to sink. All the nodes update their routing table by exchanging HELLO messages among themselves.

ii. Routing Phase: Priority based routing is proposed. For emergency data delivery highest priority is set. This is using the direct communication and hence its delay is very shorter. For normal data delivery, select a route with minimum number of hops to sink and it provides minimum delay.

iii. Scheduling Phase: TDMA slots are allocated to nodes by sink. For emergency data transmission, guaranteed time slots are given to nodes in Contention Free Period (CFP) of MAC frame. For normal data delivery, Contention Access Period (CAP) is used.

iv. Data Transmission Phase: Allocation of time slots for nodes avoids data collision. So, it reduces data packet loss.

For energy consumption with single-hop and multi-hop data communication model and path loss model, this paper is used the calculation as mentioned in equation (23), (24) and (21) respectively. Optimization function using Integer Linear Programming model has been proposed with a main objective of maximizing network lifetime. This model has been fully described in section 3.1.3.

Here, RE-ATTEMPT protocol is compared with ATTEMPT protocol. The execution of number of rounds for REATTEMPT is 1577 whereas in ATTEMPT is 1450. Comparatively the network lifetime in RE-ATTEMPT has been increased with the following reason: (a) relay nodes consume more energy than non-relay nodes, (b) unnecessary multi-hopping of data is avoided in RE-ATTEMPT, (c) alternate route selection in case of dead node, (d) hot spot detection is disabled.

Since RE-ATTEMPT protocol allows emergency data in transmission through CFP and normal data transmission through CAP, more packets are received by sink without any collision. Hence, proper scheduling using TDMA makes REATTEMPT more reliable for successful packet delivery. Therefore, packet drop ratio is decreased. If packet drop ratio decreased then the number of packets received by sink is increased. So, the throughput is considerably scaled up.

RE-ATTEMPT protocol has shown appreciably increased the lifetime of the network and throughput. Maximum lifetime has been achieved through optimization integer linear programming model. The good throughput has been attained in the course of TDMA slot allocation.

RE-ATTEMPT protocol used mathematically proven optimization model to rise the lifetime of WBAN. Considerable scheduling phase and priority based routing boosted the collision free and high speed data transmission respectively. However, more number of nodes can be deployed for testing scalability and by which lifetime of the network can be analyzed. Suppose the authors proposed an optimization model for throughput separately, still the packets reached at sink might be increased with a support of good path loss model.

M-ATTEMPT: A New Energy-Efficient Routing Protocol for Wireless Body Area Sensor Networks: The authors have proposed an M-ATTEMPT protocol [35], which is a thermal aware routing protocol for WBAN. It identifies a hot-spot node as an area that has soaring temperature due to heavy data transmission. The objective of this work is to sense the link hot-spot and routes the data away from these links. It supports the mobility of the nodes. This work dealt with the Linear programming model for maximum information extraction and minimum energy consumption.

Initialization Phase: In this phase, three different work has been executed as follows: (a). each node is informed with its neighbor, (b). position of the sink, (c). all possible routes to sink. All the nodes update their routing table by exchanging HELLO messages among themselves.

Routing Phase: Here, a path to sink with fewer hop-counts and consumes less energy is considered for regular data routing. Also, direct communication is considered for emergency data transmission. The energy consumed for single-hop and multi-hop communication is measured using the equation (23) and (24), respectively. The heating effects of the implanted sensor nodes on the human body are recognized using the Specific Absorption Rate (SAR) equation (15) and dealing with wireless communication around the human body, Specific Absorption Rate (SAR) and heating effects of the implanted sensor nodes on the human body. Whenever a node reaches a temperature threshold, then that node breaks its link with its neighbor for few rounds. As temperature returns to normal, it re-establishes the original route. This routing mechanism supports the mobility of the nodes.

Scheduling Phase: TDMA slots are allocated to nodes by the sink.

Data Transmission Phase: Allocation of time slots to nodes avoids data collision. So, it reduces data packet loss. 
The results of this protocol show good throughput with a stable network and utilize maximum energy as possible. It consumes less power and more reliable for data delivery. Also, it shows that during the mobility period, no loss of packet occurs in case of link breakage.

The placement of nodes is not according to energy levels. Hop-spot detection is a good idea to normalize its temperature. However, due to this detection, data routing is forwarded with another path. This alternate path may increase the end-to-end delay. Since authors have proposed a path with the lowest temperature until the destination is reached, there is possible for considerable power consumption, overall increasing hotness and waste of bandwidth throughout the network as most nodes are involving in routing. Moreover, it carried out low performance because of using thermal effect and mobility approach together. So, there is a need to make a network protocol robust against frequent topological changes.

SIMPLE: Stable Increased-throughput Multi-hop Protocol for Link Efficiency in WBAN: One of the significant obstacles in WBAN is to recharge the batteries. There are numerous routing protocols available for WSN, but all of them are not fit for WBAN. Sensor nodes are deployed in WBAN at fixed places. Since WBAN dealing mainly with clinical data, the critical data transmission required minimum attenuation, high reliability, and long life. Sensors transmit their data to the sink through the forwarder node. It saves the energy of nodes and network works for a more extended period.

Whenever a patient moves his hands, the wireless link of the sink with sensor nodes disconnects. Data transmission, when a link failure occurs, consumes more power of sensor nodes and relay node dropping more packets, which causes important and critical data to lose.

The objective of this protocol [36] is to provide higher throughput and more extended stability. It has eight sensor nodes and all of which are equal energy and computation capabilities. All of these nodes are known as each other's location, identity, and energy status.

SIMPLE protocol elects a new forwarder node in each round. Sink node computes the cost function described in equation (25) of all nodes. A node with a minimum cost function is preferred as a forwarder node. That is, the forwarder node has maximum residual energy and minimum distance to sink. Forwarder node assigns time slots to its children nodes for data transmission. When a node has no data to send, it switches to idle mode. Nodes wake up only at its transmission time.

Since energy is shared with all nodes by selecting a forwarder node in each round, the network lifetime, as well as residual energy, is significantly increased and network stability is maintained. So, the number of live nodes is increased. If there is a number of live nodes are available, then more packets can be transacted from one another to sink. Thus the number of packets received at the sink is increased. Since this protocol has a more extended stability period, live nodes have more cumulative path loss. Hence this protocol is useful to minimize energy consumption and to avoid hot spots.

However, the SIMPLE protocol does not consider the mobility of the nodes and not having proper optimization models for minimization of energy consumption and maximization of throughput.

iM-SIMPLE: iMproved stable increased-throughput multi-hop link efficient routing protocol for WBAN: The authors in [34] have proposed a routing protocol for minimizing energy consumption of the network and maximizing stability period, and throughput of the system are described in section 3.1.1 and 3.1.3 respectively during multi-hop mode of communication. This protocol has used the radio model, path loss model, cost function to select a forwarding node, as mentioned in equation (23), (21) and (25) respectively. Here the sink node assigns TDMA slots for forwarding node as well as regular nodes. It supports only the arm's mobility, as presented in M-ATTEMPT [35]. But the movement of nodes leads to high path loss, and therefore this action significantly reducing in throughput. The proposed smart optimization techniques worked out economically, and the resulting performance is excellent in terms of throughput, network lifetime, and energy consumption. The SIMPLE and M-ATTEMPT protocols outperform this protocol's results.

Green Communication for Wireless Body Area Networks: Energy-Aware Link Efficient Routing approach: ELR-W [3] is an energy-aware link efficient routing approach for WBANs. Energy-efficient during the routing selection process is mainly depending on the optimal utilization of the resources. The quality of the wireless link in WBANs frequently varies, which results in low reliability and energy deficiency.

This mainly focused on dynamically select the best next-hop from each Body Sensor Node (BSN) to the Body Node Coordinator (BNC) based on a number of hop counts, link efficiency, distance to BNC, and residual energy. The strength of this works is 
considering a multi-hop path having more power of intermediate nodes is considered better for energy balancing in WBAN. In this link efficiency model, each node determines its neighboring nodes according to its communication range with the use of a HELLO packet. The link efficiency between nodes is calculated as follows:

$$
L E=\sum \frac{P R_{N}}{P S_{S}}
$$

where $P R_{N}$ refers to the number of packets received at the neighbor node, and $P S_{S}$ represents the number of packets sent from the source node. The path cost function between noses is measured as follows:

$$
P C F=\sum_{\forall N_{i} \in N}\left[\alpha \times \frac{1}{R E}+\beta \times \frac{1}{L E}+\gamma \times H C+\delta \times d\right]
$$

It considers four parameters such as residual energy, link efficiency, hop-counts, distance to the $\mathrm{BNC}$ and $\alpha, \beta, \gamma$ are weighting factors for RE, LE and $\mathrm{HC}$ respectively with priority so that $\alpha+\beta+\gamma=1$. A node with least cost function is chosen as next-hop for packet forwarding. The authors created a new logic that considers link efficiency as well as shorter path for selecting the next-hop for data transmission.

The throughput and packet drops are inversely proportional to each other. The simulation result shows that ELR-W drops a lower number of packets which increases reliability. More stable energy efficiency is achieved because of using effective criteria for the selection of next-hop in the network. The proposed path cost function supports the load balancing in the network which increases throughput along with a lower number of packet drops.

Modeling induction and routing to monitor hospitalized patients in multi-hop mobility-aware body area sensor networks: This paper [12] focused on a distance-aware relaying energy-efficient (DARE) and mutual information-based DARE (MI-DARE) routing protocols for multi-hop mobility-aware BANs. Here, threshold monitoring and data monitoring sensors are used and these are equipped with limited energy resources. But main sensors and sink are having very high power sources. A mathematical model for recharging a battery of a pacemaker to monitor patients is achieved using parameters like voltage, link efficiency and quality factor. In DARE, HELLO message exchange technique is used to know each other sensors credentials. A body relay sensor is fixed on the patient's body with higher energy resources and it supports mobility. The MI-DARE routing protocol uses machine learning technique and it removes redundant data transmission. The information similarity can be identified using the probability distribution function:

$$
M I(I, J)=\sum_{i} \sum_{j} P\left(I_{i}, J_{j}\right) \log _{2}\left(\frac{P\left(I_{i}, J_{j}\right)}{P\left(I_{i}\right) P\left(J_{j}\right)}\right)
$$

Where $\mathrm{I}$ and $\mathrm{J}$ are the data at time $\mathrm{t}_{1}$ and $\mathrm{t}_{2}$ respectively. If MI value is high then $\mathrm{I}$ and $\mathrm{J}$ have similar data. If MI value is zero then I and $\mathbf{J}$ are not similar. If MI value is neither high nor zero then I and $\mathbf{J}$ are loosely related. In this way and due to mobility of nodes (communication distance reduced), energy consumption cost is reduced. It leads to prolong the network lifetime which ultimately increases the number of transmitted packets. So, DARE proves to have more throughput. But MI-DARE is not achieved throughput as much as DARE because it does not support transmitting redundant data. The nodes in DARE protocol consumes minimum energy because it uses body relays. As for as concerned about end-to-end delay, continuously increases in DARE and high in MI-DARE since MI requires more processing time. As a consequence, these two protocols are not opted for delay sensitive applications. The comparative analysis of MI-DARE, DARE and M-ATTEMPT are as follows:

\begin{tabular}{|l|l|l|l|}
\hline Metric & MI-DARE & DARE & M-ATTEMPT \\
\hline Stability Period & Highest & Moderate & Least \\
\hline Network Lifetime & Highest & Moderate & Least \\
\hline Energy Consumption & Least & Moderate & Highest \\
\hline Packet Delivery Ratio & High & High & Moderate \\
\hline
\end{tabular}




\begin{tabular}{|l|l|l|l|}
\hline End-to-End Delay & High & High & Least \\
\hline
\end{tabular}

Cluster Based Energy Efficient Routing Protocol for Wireless Body Area Network: Author has proposed cluster based energy efficient routing protocol [37] and has fixed eight different sensors nodes on the human body with same energy in homogenous mode. The Base Station (BS) is placed outside the network and rechargeable gateway is placed at the centre of the network. For clustering formation LEACH protocol is used. Here clustering model and cluster head are proposed to reduce the distance between nodes. Moreover having gateway at center of the network the distance is further reduction of transmission distance. Hence with this short nature of distance enhance the throughput and reduce the energy consumption of nodes. Also it achieved good throughput and residual energy.

Though this article has achieved reasonable result but is not recommended any new techniques or ideas to solve the existing energy consumption research issues.

Weighted Energy-Balanced Efficient Routing Algorithm for Wireless Body Area Network: This article's main intention is try to solve the hot-spots problem. A dynamic routing algorithm [11] based on multipath choosing strategy is proposed. The authors optimized the selection of path between source and destination node. This optimization could enhance the lifetime of the whole network. The path choosing strategy considers channel condition and the remaining energy of each node. Dijkshtra algorithm is used to determine a route which has a maximization of the minimum energy. As per the study of energy efficiency for WBANs, the node next to central node always becomes a hot-spot because it takes so much of forwarding tasks and data transmission. To solve this issue the improved DRA algorithm is proposed as follows:

1. Network Establishment: Initialize all nodes in the network and generate location of node $\mathrm{i}$ by $\left(\mathrm{x}_{\mathrm{i}}, \mathrm{x}_{\mathrm{j}}\right)$.

2. Calculate distance matrix according to the co-ordinates of the nodes.

3. Estimate transmission energy cost and gets the energy consumption matrix.

4. Calculate weight for each node using $W_{i}=\left(\frac{E_{i}}{k=1,2,3, \ldots, N}\right)^{-1 / r}$ where $r$ is the coefficient that adjusts the influence of the weight. Multiply the coefficient to corresponding value in the energy consumption matrix.

5. Use Dijkstra algorithm to derive one minimum energy consumption path to the central node, during which mark the nodes that are chosen as a relay node so that every node is just used only once.

Thus routing is established and starting to transmit data through the optimal path. In this algorithm weight $r$ indicates a constraint of remaining energy when node chooses the best route. The simulation result shows that when $r$ is set as smaller then the proposed routing strategy consider more factor of remaining energy. The appropriate value for $r$ is like $0.1 \leq r \leq 0.5$. The final results are shown that it preserved the node at hot spot with proper $r$ value. Hence number of live nodes with minimum energy is increased and hence life time is extended.

However this method protects the hot-spot nodes for small period of time, further transaction with sink is performed by a node which is also nearby central node. This proposal may provide a few more lifetime. But any way the node first is going to die is this kind sensor because it makes more data transmission than other.

Optimized cost effective and energy efficient routing protocol for wireless body area networks: The OCER and EOCER protocols [10] have shown all the considerations of energy dissipation for data transmission, retransmission and reception. Here probability of link reliability also measured. The OCER and E-OCER are considered for intra-BAN and inter-BAN (multi-hop communication) networks respectively. Node table and routing table are constructed to register the credentials of each node.

In order to minimize energy consumption, data followed by a node is thoroughly investigated using cost function. The following optimized cost function is framed using Genetic Algorithm to determine a optimized next hop node (forwarder node) with minimum value of cost for sending data. Three weights $w_{1}, w_{2}, w_{3}$ are considered for three parameters residual energy, path loss and link reliability in the cost function. 
Minimize:

Cost $=w_{1} \times\left|\frac{E_{\text {max }}-E_{k}(N T)}{E_{\max }}\right|+w_{2} \times\left|\frac{\operatorname{LinkR} R_{\text {max }}-\operatorname{LinkR}_{k}(N T)}{\operatorname{LinkR} R_{\max }}\right|+w_{3} \times\left|\frac{\left.P L_{\text {max }}-P L_{k} N T\right)}{P L_{\max }}\right|$ (34)

subject to:

$w_{1}+w_{2}+w_{3}=1$

Where

$0.5<w_{1}<1.0$
$0<w_{2}<1-w_{1}$
$w_{3}=1-w_{1}-w_{2}$

The authors considered three different scenarios for result analysis. In first scenario, only fixed number of packets (1000 packets) is sent and all nodes are static. Secondly, variable number of packets is sent and all nodes are static. Finally, mobility of source node with moving speed of $1 \mathrm{~m} / \mathrm{s}$ vertically is considered. As a result, for intra-BAN communication OCER protocol performed well in terms of energy consumption and for inter-BAN communication E-OCER protocol performed not up to the level since communication link is not strengthened but it is probably reliable. So, link efficiency is needed for most reliable transmission.

Even energy consumption and backside routing: An improved routing protocol for effective data transmission in wireless body area networks: The main objective of this article [7] is to extend the network lifetime, increase throughput and efficient node transition when body posture is changed. In order to achieve to increase lifetime of the network, the author has recommended minimum standard deviation value to select a forwarder node instead of just calculating a cost function mentioned in equation (25). The corresponding standard deviation function is as follows:

$\begin{aligned} \text { Standard Deviation Function }(\mathrm{i})= & \sqrt{\frac{\sum_{i=1}^{n}\left(N_{i}-m\right)^{2}}{n}} \\ & =\sqrt{\frac{\sum_{i=1}^{n}\left(N_{i}\right)^{2}}{n}}-m^{2}, i=1,2, \ldots, n,\end{aligned}$

Where $m=\frac{R E\left(N_{1}\right)+R E\left(N_{2}\right)+\ldots+R E\left(N_{n}\right)}{n}$, where $R E\left(N_{i}\right)$ is the residual energy of node $\mathrm{N}$.

Selected Path $=$ minimum [Standard Deviation Function (i) $], i=1,2, \ldots, n$. Through calculation during selection of a path to transmit data, even energy consumption of the sensors has been achieved in this article. Another different approach is adopted in routing strategy by considering sensors on the back of the body to achieve higher connection probability. Here path loss of the connection is also considered. The path loss in between two nodes at front side is $d^{3.5}$ and back side is $d^{7}$ is used. Regarding the body posture movement of the patient, the nodes shall move from signal range to another range. Suppose more parent nodes are available in the signal range, the design has to be developed by considering conditions. When a parent node reaches a maximum degree, it should not exclude the new node and decide a node to keep out based on their priority and critical need. These assumptions have been simulated and achieved an extended network lifetime, good throughput, and a regulated moved node from the communication range. Moreover, the number of sensors on both on front and backside of the body can be increased.

Energy Efficient Thermal and Power Aware (ETPA) Routing in Body Area Networks: This paper [20] is mainly devoted to minimize average temperature rise and more efficient usage of the available resources. It is a multi-hop transmission and it concentrates on individual link based on energy efficiency. A HELLO messages are sent periodically in every four frames. 
In each cycle (every four frames), each node broadcasts its temperature, available energy level through HELLO message. Suppose node $i$ becomes capable of calculating the cost of transmission to node $\mathrm{j}$ using the following equation:

$C(i, j)=\alpha_{1}\left(\frac{P_{m}-P_{i}^{j}}{P_{m}}\right)+\alpha_{2}\left(\frac{T_{j}}{T_{m}}\right)+\alpha_{3}\left(\frac{E_{m}-E_{j}}{E_{m}}\right)$

where $\alpha_{1}, \alpha_{2}$ and $\alpha_{3}$ are non-negative coefficients, $P_{i}^{j}$ is received power at node $\mathrm{i}$ from node $\mathrm{j}, P_{m}$ is maximum received power at each nodes, $T_{m}$ is representing maximum temperature allowed and $E_{m}$ is denoting maximum available energy at each nodes. Also two different assumptions are considered like packets stored for more than 2 frames must be dropped and packets which are exceeding the max_hop-count must be dropped. The following path loss model over a distance $d_{i . j}$ is considered for whole body as received signal strength from node $\mathrm{i}$ to node $\mathrm{j}$ in this work.

$$
P_{r}^{i}\left(d_{i j}\right)=P_{s}^{i}-P_{o}-10 n \log \left(\frac{d_{i j}}{d_{0}}\right)-X_{\sigma}
$$

The probability of selecting the link between node $\mathrm{i}$ and node $\mathrm{j}$ is measured using following equation:

$$
\operatorname{con}(i, j)=\prod_{\substack{w=1, w \neq i, j}}^{N} Q\left(\frac{\alpha_{2}\left(T_{j}-T_{w}\right)+\alpha_{3}\left(E_{w}-E_{j}\right)}{\alpha_{1} \sigma}\right) \times Q\left(\frac{-m_{i j}+P_{t h}}{\sigma / P_{m}}\right)
$$

where $Q(x)=\frac{1}{\sqrt{2 \pi}} \int_{-\infty}^{x} e^{\frac{-x^{2}}{2}} d x$. In this way the probability of single-hop and multi-hop communication from source to destination is determined. A single node's temperature and energy at time instant $t$ is calculated as follows:

$$
\begin{aligned}
& T_{i}^{(t+1)}=q_{i}^{(t+1)}+T_{i}^{(t)} \text { and } \\
& E_{i}^{(t+1)}=E_{i}^{(t)}-q_{i}^{(t+1)}
\end{aligned}
$$

respectively. The total energy and temperature at time to when all the packets have reached the destination is as follows:

$$
\begin{aligned}
& E_{i}^{\left(t_{0}\right)}=E_{i}^{(0)}-\sum_{j=1}^{t_{0}} q_{i}^{(j)} \text { and } \\
& T_{i}^{\left(t_{0}\right)}=T_{i}^{(0)}+\sum_{j=1}^{t_{0}} q_{i}^{(j)}
\end{aligned}
$$

respectively. In order to minimize temperature rise, the following optimization problem is proposed in this article: minimize $\max _{i} T_{i}^{\left(t_{0}\right)}$ subject to $\alpha_{1} \geq 0, \alpha_{2} \geq 0$ and $\alpha_{3} \geq 0$. This proposed routing protocol has shown significantly higher depletion time which leads to longer lasting communication and efficient usage of resources in WBAN. However the cost function is not considering the link reliability for reliable data transmission.

Designing an Energy Efficient WBAN Routing Protocol: This paper [22] is mainly concerned about Specific Absorption Rate (SAR) which is used to measure a node is crossing the limited heat energy or not. Suppose heat energy is crossed the threshold value then the patients' tissue on the location will be damaged and that particular node will not be useful to generate data or forward the data. So, the life time of the network will be decreased. This paper has studied thoroughly about 
SAR and measured SAR, for $e$ units of energy is required to transmit a single bit, then for transmission of $q$ bits, using as follows: $S A R \cong k \times q \times\left(\frac{d(e)}{d t}\right)=k \times q \times$ transmission power.

In order to act as relay/forwarder node, a node should satisfy the following inequality: $\left(\frac{E_{\text {rem }}(t-\Delta t)-E_{\text {rem }}(t)}{\Delta t}\right)^{i}<$ threshold. This threshold value is a positive value and it is corresponding SAR value not exceeding $1.6 \mathrm{~W} / \mathrm{kg}$. Energy remaining, link reliability between two nodes and node reliability are considered as a parameter to select a suitable relay node. A fitness function is also calculated to find out neighbor node as follows: $F^{j}(t)=\alpha E R^{j}(t)+\beta R_{(i, j)}(t)+\gamma R^{j}(t)+\delta\left(1-\operatorname{SARRatio}^{j}(t)\right)$,

where $\alpha, \beta, \gamma, \delta$ are weights assigned such that $\alpha+\beta+\gamma+\delta=1$. The result shown that if we assign more weight to $\mathrm{SARRatio}^{j}(t)$ than individual weight then energy is distributed between suitable forwarders. Hence this proposed scheme is used to alleviate temperature rise and increasing the network lifetime.

EAR-BAN: Energy Efficient Adaptive Routing in Wireless Body Area Networks: EAR-BAN is a centralized cluster based multi-hop routing protocol [15] proposed by combining the benefits of direct and multi-hop transmission methods. The survey of this paper reveals the fact that with increasing number of body node and decreasing distance from node coordinator, the cluster based routing approach is more energy efficient. Here on-body node with highest priority and in-body node with lowest priority is considered as default. A node distance from coordinator node is measured from signal strength of HELLO message. The node coordinator selects a node as $\mathrm{CH}$ of each cluster which has highest impact factor (IF) and minimum required energy $\left(E_{\text {cum }}\right)$ to maintain cluster operation for each round of each cluster. The IF is calculated using energy and priority of the node as follows:

$$
I F=\alpha\left(\frac{E_{i}}{E_{\max }}\right)+\beta\left(\frac{P_{i}}{P_{\max }}\right)
$$

and the minimum required energy is measured is as follows:

$E_{\text {cum }}=($ No. of cluster member) $x$ (energy consumed by a node for transmitting data in its active time slot)

If $\left(E_{\text {cum }}>E_{\text {available }}\right)$ then coordinator node chooses a nearby node as relay node to transmit data to coordinator node. Also the relay node must satisfy the condition $\left(E_{\text {available }}>=E_{\text {dependent }}\right)$. If $\left(E_{\text {cum }}<E_{\text {available }}\right)$ then $\mathrm{CH}$ directly sends data to coordinator node. If (cluster(cluster $\left.\left.\left(\mathrm{CH}\left(E_{\text {cum }}\right)\right)\right)<E_{\text {available }}\right)$ then coordinator node chooses nearby $\mathrm{CH}$ 's to transfer data towards coordinator node. The chosen $\mathrm{CH}$ must satisfy the condition $\left.\left(E_{\text {available }}\right\rangle=E_{\text {dependent }}\right)$. The simulation result of this work shows the fact that uniform distribution of body nodes prolonged the network lifetime when coordinator node is placed at the centre of the human body.

A low overhead tree-based energy-efficient routing scheme for multi-hop wireless body area networks: Using fixed power levels to send packets either wastes energy or fails to meet data transmission. Since the quality of link is changing dynamically, it is great challenge to predict it in WBAN and this algorithm selects the transmission power to ensure that sensors transmit packets with enough power. So, this paper [8] considering jointly adaptive power control and routing in multi-hop WBANs. This work has three modules like route selection, link quality estimation and data forwarding.

The total energy consumption of node $n$ with different power levels with packets forwarded by and originally transmitted by node $i$ are calculated using following equation:

$$
C_{i}=\sum_{l=1}^{L} C_{i, l}
$$

where $i \in\{1,2,3, \ldots, N\}$ and $l \in\{1,2,3, \ldots, L\}$,

$C_{i, j}=w_{l}\left(m_{i, l}^{R}+m_{i, l}^{S}\right)$. 
This mechanism is to adopt a power levels of node $i$ according to link quality. To meet the QoS requirement a node may use high power level. To measure the transmission quality (cost) energy-aware expected transmissions (eETX) is used. Since this protocol is tree based architecture, expected transmissions (ETX) is used to show the link quality between a node and its parent node. In this work transmission quality is represented by link quality and energy consumption states. Here, link eETX between a node $i$ and its neighbor $\bar{i}$ at power level 1 is defined as follows:

$$
\bar{q}_{l}(i, \bar{i})=w_{l} p_{l}(i, \bar{i})+\alpha\left(\frac{C_{\bar{i}}}{\sum_{i^{\prime} \in I^{\prime}} C_{n^{\prime}}}\right) .
$$

If $\alpha$ is very small the link quality is good and if $\alpha$ is very large link quality is weak. Also this paper has defined the path eETX of a sensor node as the path eETX of its neighbor plus the eETX of its link to its neighbor. Therefore the path eETX of node $i$ is denoted as follows: $q(i, s)=\bar{q}_{l}(i, \bar{i})+q(\bar{i}, s)$. This power-adaptive routing algorithm's aim is to find the path from node $\mathrm{i}$ to sink s with lowest path eETX as parent node and it is expressed as $q^{*}(i, s)=\min _{l, \bar{i}}\left(\bar{q}_{l}(i, \bar{i})+q(\bar{i}, s)\right.$. In order to find $q^{*}(i, s)$, this scheme is adaptively choosing relay and power levels. Each node sends HELLO message containing the values of $\bar{q}_{l}(i, \bar{i}), 1$ and $C_{i, l}$ to its neighbors. The experimental detail has considered three different body postures like standing (static), walk (3 Km/hr) and run $(7 \mathrm{Km} / \mathrm{hr})$. The transmission power is supported by with four levels such as $0 \mathrm{dBm},-5 \mathrm{dBm},-10 \mathrm{dBm}$ and $-15 \mathrm{dBm}$. This works focused on four different mechanisms in order to equip the proposed algorithm as robust against for any kind of challenges. The simulation result has shown that EERS is achieved a good tradeoff between reliability, delay and energy consumption.

An Energy Efficient Fuzzy based Adaptive Routing Protocol for Wireless Body Area Network: This paper [16] considered both direct message passing and cluster based technique. The fuzzy logic concept is used for selecting a $\mathrm{CH}$ according to node priority, node deployment density, remaining energy and distance with coordinator node. Here maximum transmission range is $100 \mathrm{~cm}$. If the priority of the node is 0.5 then it is treated as implanted node and if the priority of the node is 1 then it is considered as on-body critical node. The only non-critical nodes are clustered based on their impact factor (IF), distance node's deployment density. The IF is defined as follows:

$$
I . F=\frac{R E(i)}{E_{0}}+\frac{P_{i}}{P_{0}}
$$

and it requires two parameters such as energy and priority of a node. The probability of each node to become a $\mathrm{CH}$ is the output of fuzzy Inference system. For every cluster, the average distance of all the cluster members to its $\mathrm{CH}$ is calculated as follows:

$$
D_{m, c k}=\frac{\sum_{i=1}^{N} D_{C H}(i)}{N},
$$

where $D_{C H}(i)$ is the distance of $\mathrm{i}^{\text {th }}$ cluster member to its $\mathrm{CH}$ and $\mathrm{N}$ is the number of cluster members in a cluster. The nearest node of the given node is selected if a node satisfies the following two conditions: $D_{m, c k}<D_{m}$ and $D_{m, c k}<D_{i}$. Where $D_{m}=\frac{\sum_{i=1}^{N} P_{B N C}(i)}{N}$ and $D_{i}$ is the distance of the $\mathrm{i}^{\text {th }}$ cluster member from the BNC. To avoid the congestion and loss of data packets, TDMA time slots are used. $\mathrm{CH}$ is being changed in each and every round to distribute the energy consumption among all the nodes. The $\mathrm{CH}$ node should satisfy the condition that remaining energy of a node greater than minimum threshold energy of a node. This protocol obtained considerable stability period and network lifetime. Also transmits more packets.

Modified New-Attempt Routing Protocol for Wireless Body Area Network: HELLO message is used to spread a node's information to nearby nodes and all the nodes update their routing table accordingly. It uses both multi-hop 
communication and for emergency purpose uses direct communication. This protocol [18]selects a new forwarder node using the following equation in each and every round in order to equally distribute the energy among the nodes.

$$
C F(i)=\frac{R E(i)}{d^{*} q}
$$

where $\mathrm{d}$ is the distance between sink and a node $\mathrm{i}, \mathrm{q}$ represents average data rate of all nodes. The cost function with maximum value is chosen as a forwarder node. Residual energy, network lifetime, path loss and throughput are the performance metrics considered to analyze the working level of the protocol.

ESR: Energy aware and Stable Routing protocol for WBAN networks: The objective of this paper [17] is to select energy efficient path with stable link to reach stable coordinator. A new path established from source node to destination node with multi-hop distance using route request (RREQ) and response (RREP) messages. While RREQ and RREP is flowing on either direction, the concerned nodes routing tables are updated. The best path is selected from source to destination based on energy consumption and path stability. In order to reach this path this paper has proposed two different function such as energy aware function and link stability aware function are defined. The energy cost function of node $\mathrm{i}$ belonging to the path $\mathrm{j}$ denoted by $f e n_{i, j}(t)$ and defined as follows:

$$
\begin{aligned}
& f e n_{i, j}(t)=\frac{\operatorname{Elev}_{i, j}(t)}{D R_{i, j}(t)} \times w_{i, j} \\
& \text { where } \operatorname{Elev}_{i, j}(t)=\frac{E_{i, j}(t)}{E_{\text {average }}}
\end{aligned}
$$

and $\mathrm{DR}_{\mathrm{i}, \mathrm{j}}(\mathrm{t})$ is the rate at which energy is consumed at a given node. The minimum residual energy of nodes making a route $\mathrm{j}$ form source to destination is measured as follows:

$$
f e p_{j}(t)=\min _{i=1}^{n-1}\left(\operatorname{fen}_{i, j}(t)\right)
$$

The stability cost function of the link from $\mathrm{i}$ to $\mathrm{k}$ at time $\mathrm{t}$ is given as follows:

$f_{s} l_{i, j}(t)=\frac{S D l_{i, k}(t)}{M l_{i, k}(t)}$ where mean of $\mathrm{m}$ distances recorded between nod $\mathrm{I}$ to $\mathrm{k}$ is represented as follows:

$$
M l_{i, k}(t)=\frac{\sum_{t=t 1}^{t m} d_{i, k}(t)}{n}
$$

and standard deviation of the distances recorded between the node i to $\mathrm{k}$ is measured as follows:

$$
S D l_{i, k}(t)=\sqrt{\frac{1}{n} \sum_{t=t 1}^{t n}\left(d_{i, k}(t)-M l_{i, k}(t)\right)^{2}} .
$$

The path cost function stability is the maximum stability cost of the links constituting a path and it is defined as follows:

$$
f s p_{j}(t)=\max _{i=1}^{n-1}\left(f s l_{i, k}(t)\right)
$$


where $\mathrm{n}$ is the nodes number of path from $\mathrm{j}$ to $\mathrm{k}$ as the neighbor node of the node $\mathrm{i}$. If $f s p_{j}(t)$ tends to zero then the link is stable else if $f s p_{j}(t)$ tends to one/infinity then the link is unstable. Therefore this paper has proposed a path selection principle

by combining the energy consumption and link stability of a path from node $\mathrm{i}$ to $\mathrm{j}$. The path model has defined as follows:

$$
f p_{j}(t)=\alpha f e p_{j}(t)+\frac{\beta}{f s p_{j}(t)}
$$

where $\alpha+\beta=1$. A path with maximum $f p_{j}(t)$ is chosen to forward the data packets. The simulation result has shown clearly that this model improves the network performance, consumes less energy and minimizes routing overhead traffic. Also it improves the mobile WBAN network performance.

Trust and Thermal Aware Routing Protocol (TTRP) for Wireless Body Area Networks [23]: Trust is a calculation that can be obtained by observing the packet forwarding behavior of relay node and is a continuous fractional value between 0 and 1. If trust value of a node is less than 0.5 then that node is untrusted node, and if trust value is greater than 0.6 then that node is treated as trusted relay node. The trust is divided into two categories like direct-trust and indirect-trust. The total trust of a node is obtained using the following equation:

$$
T_{R i, R j}(t)=\alpha D T_{R i, R j}(t)+\beta \frac{I T_{R i, R j}^{R k}}{N_{R j}}(t)
$$

Here the direct trust $\mathrm{DT}_{\mathrm{Ri}, \mathrm{Rj}}$ indicates that direct trust of relay node $\mathrm{Ri}$ over $\mathrm{Rj}$ at time $\mathrm{t}$ and $I T_{R i, R j}^{R k}$ represents the indirect trust of relay node $\mathrm{Ri}$ over $\mathrm{Rj}$ obtained through $\mathrm{Rk}$ at time t. The weights $\alpha$ and $\beta$ are assigned to direct and indirect trust respectively where $\alpha+\beta=1$ and $\alpha>\beta \cdot N_{R j}$ represents all neighboring relay nodes of $\mathrm{Rj}$.

The motivation of temperature aware routing is to distribute traffic load and therefore minimize number of nodes becomes a hotspot. The following composite function (CF) balances traffic load in the network based on temperature and it selects all the trusted nodes along the routes. $C F=(w 1 *$ Trust $)+(w 2 *$ Temp $)$ where w1 and w2 are weights of trust and temperature respectively and $w 1+w 2=1$. Suppose a relay node reaches a threshold value then it is marked as hotspot node by its neighboring node. Therefore a route with minimum cost will be selected for relay data packets to gateway node.

When threshold value of temperature exceeds and/or trust value of neighboring node is below the threshold the link failure may occur. This could be handled by this protocol in maintenance phase. Therefore TTRP is protecting against malicious nodes and avoid the hotspot issue. Including of weighting factors of temperature and trust minimizes the evolving of hotspots and finding new routes.

\section{WBAN and Other Networks}

Compared to other Wireless Networks scalability, WBAN is restricted to the limited coverage area and low density of nodes. Moreover, WBAN uses maximum star-based centralized topology and standard of WBAN defined in terms of using communicating technologies. Table I describes the comparison between WBAN with other wireless technologies.

\begin{tabular}{|c|c|c|c|c|}
\hline Properties/Networks & $\begin{array}{l}\text { Wireless Sensor } \\
\text { Networks }\end{array}$ & $\begin{array}{l}\text { Wireless Body Area } \\
\text { Networks }\end{array}$ & MANET & VANET \\
\hline Scalability & $\begin{array}{ll}\text { Large } & \text { area } \\
\text { coverage }(\mathrm{m})\end{array}$ & $\begin{array}{l}\text { Limited to the human } \\
\text { body }\end{array}$ & $\begin{array}{lll}\begin{array}{l}\text { Large } \\
(\mathrm{Kms})\end{array} & \text { area coverage } \\
\end{array}$ & $\begin{array}{l}\begin{array}{l}\text { Wide area coverage } \\
(\mathrm{Kms})\end{array} \\
\end{array}$ \\
\hline Deployment Density & $\begin{array}{ll}\text { very } & \text { densely } \\
\text { deployed } & \\
\end{array}$ & Low Density & $\begin{array}{l}\text { Based on mobility of } \\
\text { nodes }\end{array}$ & $\begin{array}{l}\text { Based on mobility of } \\
\text { nodes }\end{array}$ \\
\hline $\begin{array}{l}\text { Data rate and } \\
\text { Frequency }\end{array}$ & $54 \mathrm{Mbps} \& 2.4 \mathrm{Ghz}$ & $\begin{array}{l}\text { 10Mbps } \\
\text { On-body :3.1-1.6 Ghz } \\
\text { In-plant :402-405Mhz }\end{array}$ & $11 \mathrm{Mbps} \& 2.4 \mathrm{Ghz}$ & 27Mbps \&5.9Ghz \\
\hline Network & Star/mesh & Star & Peer-to-peer /Star & Peer-to-peer /Star \\
\hline
\end{tabular}

Table I: Comparison WBAN and other Networks 


\begin{tabular}{|l|l|l|l|l|}
\hline topology & \multicolumn{1}{|l|}{} & & \\
\hline Energy Scavenging & $\begin{array}{l}\text { Solar or wind } \\
\text { power }\end{array}$ & Motion or body heat & Solar or wind power & Vehicle battery \\
\hline Mobility & No mobility & Body Movement & High & Very high \\
\hline $\begin{array}{l}\text { Standards and } \\
\text { Technologies }\end{array}$ & $\begin{array}{l}\text { WEE 802.11 \& } \\
\text { WiFi }\end{array}$ & $\begin{array}{l}\text { IEEE 802.15.6 } \\
\text { zigbee/Bluetooth/WiFi }\end{array}$ & IEEE 802.11\& $\& 02.11 \mathrm{p}$ & $\begin{array}{l}\text { IEEE } \\
\text { DSRC,WAVE }\end{array}$ \\
\hline $\begin{array}{l}\text { Change in Network } \\
\text { Topology }\end{array}$ & No No & slow & Frequently and fast \\
\hline
\end{tabular}

\section{Conclusion and Future directions}

This paper has discussed different energy-efficient routing protocols concerning temperature based, cluster-based, costeffective based, link-aware based, and QoS based protocols. We have demonstrated the structure of the sensor unit and the types of WBANs. This paper has analyzed and described a linear integer optimization approach for maximization of network lifetime and throughput and, minimization of energy consumption of WBAN. The comparison of WBANs and other wireless networks technologies are given and, research opening in WBAN also presented and also dealt with path loss model to increase throughput, radio model for communication and radiation energy analysis model to reduce the hot-spot which affect the human tissues. All the discussed protocols of this paper, irrespective of their special interest, mainly considered to select a forwarder node with minimum cost function value and maximum energy of the node. This literature found that for normal and emergency data communication, the used communication model is multi-hop and single-hop, respectively. The temperature-based routing protocols are mainly concerned about hot-spot regions. These regions are avoided or data packets are re-routed to reach the sink. The cluster and cross-layer based protocols are taking care of the connectivity of nodes to disseminate the data. Since WBAN is concerned about patients' data, connectivity among the nodes, and security of data are very important. QoS routing protocols are taking care of the reliability of data.

We consider that a routing protocol in WBANs aim is to reduce the heat energy and send the data as soon as possible by selecting minimum cost function with maximum energy nodes on the path. The safety of the human body is the most important than other things. So, energy-efficient routing protocol should be taken as the primary objective of WBAN, among other metrics.

Future routing protocols for WBANs should be considered to receive the QoS and reliable data. So, the security of the data needs to be considered. To transfer the data efficiently, link capacity and quality need to be upgraded. By deploying more number of bio-sensors in the human body, hot-spot nodes may be avoided. This could be done by designing the efficient energy utilized routing protocol is needed. So, the tissues of the human body will not be affected.

Declaration: We author of the above titled paper hereby declare that the work included in the above paper is original and is an outcome of the research carried out by the authors indicated in it. Further, We author declare that the work submitted for Wireless Personal Communications An International Journal has not been published already or under consideration for publication in any Journals/Conferences/Symposia/Seminars.

Funding (Not Applicable)

Conflicts of interest/Competing interests (Not Applicable)

Availability of data and material (data transparency) (Not Applicable)

Code availability (software application or custom code) (Not Applicable)

\section{REFERENCES}

[1]. NingWang et al. (2017). A cross-layer approach to message authentication based on sparse representation for wireless body area networks. International Journal of Distributed Sensor Networks, Vol. 13(3).

[2]. Neelam Sharma et al. (2018). An enhanced-simple protocol for wireless body area networks. Journal of Engineering Science and Technology Vol. 13, No. 1, $196-210$.

[3]. Muhammad Anwar et al.(2018). Green Communication for Wireless Body Area Networks: Energy Aware Link Efficient Routing Approach. Sensors. 18, 3237. DOI:10.3390/s18103237.

[4]. R. Srinivasan et al. (2018) "Energy Harvesting Based Efficient Routing Scheme for Wireless Sensor Network", Wireless Personal Communications: An International Journal. Volume 101, Issue 3, pp 1457-1468

[5]. Bahae Abidi.(2018). An energy efficiency routing protocol for wireless body area networks. Journal of Medical Engineering \& Technology. Vol. 42(4):290-297. 
[6]. J. Naskath et al. (2018). Location optimization for road side unit deployment and maximizing communication probability in multilane highway. International Journal of Heavy Vehicle Systems, Vol. 25, Nos. 3/4.

[7]. Ilkyu Ha et al. (2016). Even energy consumption and backside routing: An improved routing protocol for effective data transmission in wireless body area networks. International Journal of Distributed Sensor Networks. Vol. 12(7) 1-11.

[8]. Liang Liang et al. (2014). A low overhead tree-based energy-efficient routing scheme for multi-hop wireless body area networks. Journal of Computer Networks. Volume 70, Pages 45-58.

[9]. Jocelyne Elias et al. (2014). Optimal design of energy-efficient and cost-effective wireless body area networks. Elsevier Ad Hoc Networks Vol.13, 560-574.

[10]. Navneet Kaur et al. (2017). Optimized cost effective and energy efficient routing protocol for wireless body area networks. Elsevier Ad Hoc Networks Vol. 61, 65-84.

[11]. Zhuoming Li et al. (2016). Weighted Energy-Balanced Efficient Routing Algorithm for Wireless Body Area Network. International Journal of Distributed Sensor Networks. Article ID 7364910. 7 pages.

[12]. Nadeem Javaid et al. (2016). Modeling induction and routing to monitor hospitalized patients in multi-hop mobility-aware body area sensor networks. EURASIP Journal on Wireless Communications and Networking. DOI 10.1186/s13638-016-0643-z.

[13]. Ashfaq Ahmad et al. (2014). RE-ATTEMPT: A new energy-efficient routing protocol for wireless body area sensor networks. International Journal of Distributed Sensor Networks. Article ID 464010, 9 pages DOI: 10.1155/2014/464010.

[14]. Lin Li et al. (2018). An Energy-Balanced Routing Protocol for a Wireless Sensor Network. Journal of Sensors. Article ID 8505616, 12 pages DOI 10.1155/2018/8505616.

[15]. Md. Tanvir Ishtaique ul Huque et al. (2013). EAR-BAN: Energy Efficient Adaptive Routing in Wireless Body Area Networks. IEEE International Conference on Signal Processing and Communication Systems (ICSPCS)

[16]. Kshitiza Singh et al. (2015). An Energy Efficient Fuzzy based Adaptive Routing Protocol for Wireless Body Area Network. IEEE UP Section Conference on Electrical Computer and Electronics (UPCON).

[17]. Omar Smail et al. (2016). ESR: Energy aware and Stable Routing protocol for WBAN networks. International Wireless Communications Mobile Computing Conference (IWCMC).

[18]. Smita Singh et al. (2016). Modified New-Attempt Routing Protocol for Wireless Body Area Network. International Conference on Advances in Computing, Communication, \& Automation (ICACCA).

[19]. Jyoti Anandet et al. (2017). Comparative analysis of energy efficient routing in WBA. IEEE International Conference on Computational Intelligence and Communication Technology (IEEE-CICT).

[20]. Samaneh Movassaghi et al. (2012). Energy efficient thermal and power aware (ETPA) routing in Body Area Networks. IEEE International Symposium on Personal, Indoor and Mobile Radio Communications (PIMRC).

[21]. Priya Juneja et al. (2015). Tree Based Energy Efficient Routing Scheme for Body Area Network. IEEE International Conference on Advances in Computer Engineering and Applications (ICACEA).

[22]. Moumita Roy et al. (2017). Designing an Energy Efficient WBAN Routing Protocol. IEEE International Conference on Communication Systems and Networks (COMSNETS).

[23]. Ali Raza Bhangwar et al. (2017). Trust and Thermal Aware Routing Protocol (TTRP) for Wireless Body Area Networks. Wireless Personal Communications: An International Journal Vol.97:349-364.

[24]. Rahat Ali Khan et al. (2018). An Energy Efficient Routing Protocol for Wireless Body Area Sensor Networks. Wireless Personal Communications: An International Journal. DOI: 10.1007/s11277-018-5285-5.

[25]. Maryam Asgari et al. (2015). Overview of routing algorithms in WBAN. Advances in Computer Science: an International Journal. Vol. 4, Issue 4, No.16 14-20.

[26]. V. Bhanumathi et al. (2017). A guide for the selection of routing protocols in WBAN for healthcare applications. Springer Human Centric Computing and Information services. 7:24 DOI 10.1186/s13673-017-0105-6.

[27]. Samaneh Movassaghi et al. (2013). A Review of Routing Protocols in Wireless Body Area Networks", Journal of Networks, Vol. 8, NO. 3.

[28]. Haibat Khan et al. (2018). Highly Efficient Privacy-Preserving Key Agreement for Wireless Body Area Networks. IEEE International Conference On Trust, Security And Privacy In Computing And Communications.

[29]. Christian Henry Wijaya Oey et al. (2013). A Survey on Temperature-Aware Routing Protocols in Wireless Body Sensor Networks. Sensors 13(8). 9860-77.

[30]. Zheng Zhang et al. (2015). A survey of sparse representation: algorithms and applications. IEEE Access. Vol. 3, 490-530.

[31]. V. Kavidha et al. (2018). Novel energy-efficient secure routing protocol for wireless sensor networks with Mobile sink. Peer-to-Peer Networking and Applications. DOI: 10.1007/s12083-018-0688-3.

[32]. Samaneh Movassaghi et al. (2014). Wireless Body Area Networks: A Survey. IEEE Communications Surveys \& Tutorials. DOI: 10.1109/SURV.2013.121313.00064.

[33].https://www.un.org/development/desa/publications/world-population-prospects-th2017-revision.html 
[34]. Nadeem Javaid et al. (2015). iM-SIMPLE: iMproved stable increased-throughput multi-hop link efficient routing protocol for Wireless Body Area Networks. Journal of Computers in Human Behavior (Elsevier). 1003-1011.

[35]. N. Javaid et al. (2013). M-ATTEMPT: A New Energy-Efficient Routing Protocol for Wireless Body Area Sensor Networks. The $4^{\text {th }}$ International Conference on Ambient Systems, Networks and Technologies (ANT 2013). Procedia Computer Science 19 (2013). 224-231.

[36]. Q. Nadeem et al. (2013). SIMPLE: Stable Increased-throughput Multi-hop Protocol for Link Efficiency in Wireless Body Area Networks. IEEE International Conference on Broadband and Wireless Computing, Communication and Applications (BWCCA'13).

[37]. Turki Ali Alghamdi (2016) Cluster Based Energy Efficient Routing Protocol for Wireless Body Area Networks. Trends in Applied Sciences Research (Academic Journals Inc). Vol 11(1). 12-18

[38]. Hayajneh, T, Almashaqbeh, G, Ullah, (2014) S. A survey of wireless technologies coexistence in WBAN: analysis and open research issues. Wire Networks; 20(8): 2165-2199.

[39]. Saarika, U, Sharma, PK, Sharma, D (2016). A roadmap to the realization of wireless body area networks: a review. In: Proceedings of the international conference on electrical, electronics, and optimization techniques (ICEEOT), Chennai, India, 35 March. New York: IEEE.

[40]. Pantelopoulos, A, Bourbakis, NG (2010). A survey on wearable sensor-based systems for health monitoring and prognosis. IEEE T Syst Man Cy C; 40(1): 1-12.

[41]. Lai, X, Liu, Q, Wei, X. A survey of body sensor networks. Sensors 2013; 13(5): 5406-5447.

[42]. Negra, R, Jemili, I, Belghith (2016), A. Wireless body area networks: applications and technologies. Procedia Comput Sci; 83: $1274-1281$.

[43].A Bag, MA Bassiouni (2008). Routing algorithm for network of homogeneous and id-less biomedical sensor nodes (RAIN). Sensors Applications Symposium. SAS 2008. IEEE. 68-73.

[44] N Javaid, Z Abbas, M Fareed, Z Khan, N Alrajeh. M-ATTEMPT(2013): A new energy-efficient routing protocol for wireless body area sensor networks. Procedia Computer Science. 19: 224-231.

[45] S Hu, J Han, X Wei, Z Chen.(2015) A multi-hop heterogeneous cluster-based optimization algorithm for wireless sensor networks. Wireless Networks; 21: 57-65.

[46] S Ahmed, N Javaid, S Yousaf, A Ahmad, M Sandhu, M Imran, et al.(2015) Co-LAEEBA: Cooperative link aware and energy efficient protocol for wireless body area networks. Computers in Human Behavior.

[47]. Abdullah, WAN, Yaakob, N, Elobaid, ME(2016). Energy-efficnergy-efficient remote healthcare monitoring using IoT: a review of trends and challenges. In: Proceedings of the international conference on Internet of Things and cloud computing, Cambridge,. New York: ACM.

[48]. Khan, RA, Memon, S, Zardari, S (2016). Transposition technique for minimization of path loss in wireless on-body medical sensors. Sindh Univ Res J; 48(4): 747-754.

[49]. Majumder, AB, Gupta (2018), S. An energy-efficient congestion avoidance priority-based routing algorithm for body area network. lecture notes in networks and systems, vol. 11. Singapore: Springer.

[50]. Yang, X, Wang, L, Zhang, Z (2018). Wireless body area networks MAC protocol for energy efficiency and extending lifetime. IEEE Sensors Lett; 2(1): 1-4.

[51]. Leeand, J, Kim, S (2018). Emergency-prioritized asymmetric protocol for improving QoS of energy-constraint wearable device in wireless body area networks. Appl Sci 8(1): 92

[52]. Samantaand, A, Misra (2018), S. Energy-efficient and distributed network management cost minimization in opportunistic wireless body area networks. IEEE Transactions Mobile Computing 2018; 17(2): 376-389.

[53]. Altun, A., \& Bascifci, N. (2011). A wireless sensor network based on zigbee for ECG monitoring system. In Application of Information and Communication Technologies (AICT), (pp. 1-5). IEEE. doi:10.1109/ICAICT.2011.6111002

[54]. Ababneh, N., Timmons, N., Morrison, J., \& Tracey, D. (2012). Energy-balanced rate assignment and routing protocol for body area networks. In Advanced Information Networking and Applications Workshops (WAINA), pp. 466-471. IEEE. doi:10.1109/WAINA.2012.34.

[55]. Liu J, Chen Y, Wang Y, Chen X, Cheng J, et al. (2018) Monitoring vital signs and postures during sleep using Wi-Fi signals. In IEEE Internet of Things Journal 5(3): 2071-2084.

[56]. Latre B, Bart B, Ingrid M, Chris B, Elisabeth R, et al. (2007) A low-delay protocol for multihop wireless body area networks. 4th Annual International Conference on Mobile and Ubiquitous Systems: Networking \& Services (MobiQuitous), Philadelphia, USA, pp. 1-8.

[57]. M Ameen, A Nessa, KS Kwak (2008). QoS issues with focus on wireless body area networks. Convergence and Hybrid Information Technology. ICCIT'08.PP. 801-807. 
[58]. Rahim, N Javaid, M Aslam, U Qasim, Z Khan (2012). Adaptive-reliable medium access control protocol for wireless body area networks. Sensor, Mesh and Ad Hoc Communications and Networks, Annual IEEE Communications Society, PP: 56-58

[59]. Braem, B, Latre, B, Moerman, I(2006), The wireless autonomous spanning tree protocol for multihop wireless body area networks., IEEE, Proceedings of the 2006 third annual international conference on mobile and ubiquitous systems: networking \& services, San Jose, CA, 17-21.

[60]. Nadeem, Q, Javaid, N, Mohammad (2013), SN. Simple: stable increased-throughput multi-hop protocol for link efficiency in wireless body area networks. IEEE, Proceedings of the eighth international conference on communication and applications (BWCCA) broadband and wireless computing.

[61]. Yating Qu , Guoqiang Zheng, Honghai Wu, Baofeng Ji and Huahong Ma (2019) An Energy-Efficient Routing Protocol for Reliable Data Transmission in Wireless Body Area Networks, Sensors, 19, 4238; doi:10.3390/s19194238.

[62]. Bogdan Antonescu, Stefano Basagni (2013) Wireless Body Area Networks: Challenges, Trends and Emerging Technologies, DOI 10.4108/icst.bodynets.2013.253722.

[63]. Ilkyu Ha (2015) Technologies and Research Trends in Wireless Body Area Networks for Healthcare: A Systematic Literature Review, International Journal of Distributed Sensor Networks, Volume: 11 issue: 6.

[64]. Rahat Ali Khan, Al-Sakib Khan Pathan (2018) The state-of-the-art wireless body area sensor networks: A survey, International Journal of Distributed Sensor Networks, https://doi.org/10.1177/1550147718768994. 